\title{
Metabolic Physiological Networks: The Impact of Age
}

\author{
Antonio Barajas-Martínez 1,2,3, Jonathan F. Easton 2,4, Ana Leonor Rivera', \\ Ricardo Martínez-Tapia ${ }^{1,3}$, Lizbeth de la Cruz ${ }^{1}$, Adriana Robles-Cabrera ${ }^{3,4}$ and \\ Christopher R. Stephens ${ }^{2,4 *}$
}

${ }^{1}$ Department of Physiology, School of Medicine, Universidad Nacional Autónoma de México, Mexico City, Mexico, ${ }^{2}$ Centro de Ciencias de la Complejidad, Universidad Nacional Autónoma de México, Mexico City, Mexico, ${ }^{3}$ Programa de Doctorado en Ciencias Biomédicas, Universidad Nacional Autónoma de México, Mexico City, Mexico, ${ }^{4}$ Instituto de Ciencias Nucleares, Universidad Nacional Autónoma de México, Mexico City, Mexico

Metabolic homeostasis emerges from the interplay between several feedback systems that regulate the physiological variables related to energy expenditure and energy availability, maintaining them within a certain range. Although it is well known how each individual physiological system functions, there is little research focused on how the integration and adjustment of multiple systems results in the generation of metabolic health. The aim here was to generate an integrative model of metabolism, seen as a physiological network, and study how it changes across the human lifespan. We used data from a transverse, community-based study of an ethnically and educationally diverse sample of 2572 adults. Each participant answered an extensive questionnaire and underwent anthropometric measurements (height, weight, and waist), fasting blood tests (glucose, HbA1c, basal insulin, cholesterol HDL, LDL, triglycerides, uric acid, urea, and creatinine), along with vital signs (axillar temperature, systolic, and diastolic blood pressure). The sample was divided into 6 groups of increasing age, beginning with less than 25 years and increasing by decades up to more than 65 years. In order to model metabolic homeostasis as a network, we used these 15 physiological variables as nodes and modeled the links between them, either as a continuous association of those variables, or as a dichotomic association of their corresponding pathological states. Weight and overweight emerged as the most influential nodes in both types of networks, while high betweenness parameters, such as triglycerides, uric acid and insulin, were shown to act as gatekeepers between the affected physiological systems. As age increases, the loss of metabolic homeostasis is revealed by changes in the network's topology that reflect changes in the system-wide interactions that, in turn, expose underlying health stages. Hence, specific structural properties of the network, such as weighted transitivity, i.e., the density of triangles in the network, can provide topological indicators of health that assess the whole state of the system. Overall, our findings show the importance of visualizing health as a network of organs and/or systems, and highlight the importance of triglycerides, insulin, uric acid and glucose as key biomarkers in the prevention of the development of metabolic disorders.

\footnotetext{
Keywords: metabolic syndrome, physiological networks, systems biology, biomarkers, aging
} 


\section{INTRODUCTION}

Metabolic homeostasis arises from the interchanges between multiple chains of biochemical reactions and their mechanical responses. These exchanges maintain variables related to energy expenditure and energy availability within suitable ranges for the organism. The components of these chains are shared by multiple others, thereby constituting a metabolic network. Unfortunately, many processes of this network are not readily accessible in the clinical setting. Therefore, to make inferences about the underlying energy metabolism, various biomarkerseither biochemical or anthropometric-have been used to assess the state of the different physiological sub-systems that constitute the network. These physiological variables represent either regulated variables or physiological response systems (Fossion et al., 2018). The lability of the values of physiological response variables, and the consequent stability of regulated variables, characterizes the robustness of a complex homeostatic system that resorts to pathological states only in order to preserve vital variables (Kitano et al., 2004). Thus, homeostasis can be established by the interplay between physiological variables, allowing its study through a metabolic physiological network.

Over time, the physiological compensatory systems that maintain homeostasis become worn down due to the cumulative impact of metabolic insults, transitioning from healthy to maladaptive states that precede disease onset (Stephens et al., 2020). An already existing medical notion of this systemwide progression of states before the overt onset of disease is metabolic syndrome (MetS), whose prevalence increases strongly with age (Hildrum et al., 2007) and unhealthy lifestyles. At early stages, MetS biomarkers indicate invisible alterations, wherein homeostasis can still be preserved (Huang, 2009). Insulin resistance, dyslipidemia, endothelial dysfunction, prothrombotic, proinflammatory states and, more recently, oxidative stress are then employed to diagnose a condition of increased cardiometabolic risk (Reaven, 1993; Vona et al., 2019). With this in mind, several medical organizations established operational diagnostic criteria ( $\mathrm{Xu}$ et al., 2018), starting with preexisting diagnostic thresholds for each associated disease, and then lowering them in order to provide a preventive focus for the diagnosis of MetS (Parikh and Mohan, 2012). In the continued presence of metabolic insults, as each physiological regulatory system fails, the cascade is absorbed downstream by the next system. Eventually, what was originally reversible pathological states progress to become irreversible diseases. This is the final stage, characterized by the lability of the regulated variables, wherein the physiological response systems become overwhelmed. These states correspond to clinical diseases that were the basis for the first historical descriptions of MetS, where gross anatomical changes and clinically overt symptoms, comprising obesity, hypertension, gout, atherosclerosis and obstructive apnea were first associated (Enzi et al., 2003). However, it is usually on a scale of decades that these physiological interactions change substantially. Disease appears only once the robustness of the metabolic physiological network is broken, and regulated variables lose their tight control.
The current approach to determining metabolic health relies on using the thresholds of individual biomarkers, without considering the overall physiological network itself. As threshold values are the result of a compromise between sensitivity and specificity, they must be tailored adequately for both screening and diagnostic purposes in each population (Almeda-Valdes et al., 2016). However, current thresholds consider neither age stratification nor the duration of the pathological states, resulting in medical interventions that are targeted toward single variables and only late in life (Easton et al., 2019). Furthermore, standard of care for these complex states is no different from the treatment of each of its individual components (Kahn, 2007). Although targeted approaches for age have been proposed, for providing further insight on the etiology of risk factors and guide diseaseprevention strategies (Leventhal et al., 2014; Leatherdale, 2015; $\mathrm{Xu}$ et al., 2018), it has been argued that the principle utility of MetS as a concept relies on the preventive nature of its scope, and the idea that single interventions could improve simultaneously all of the current five MetS criteria (Vassallo et al., 2016). However, there is still doubt as to how to weight the risk associated with each factor, or their combinations (Sattar, 2008). Indeed, given the increasing abundance of metabolic biomarkers that predict disease, there is not even a universal consensus on which criteria should be included and excluded in the first place in order to best assess metabolic health (O'Neill and O'Driscoll, 2015). As metabolic health is an emergent property, arising from the interaction of multiple physiological systems over time, the framework of complexity provides the means for a wholesystem analysis (Lusis et al., 2008; Haring et al., 2012; Sun et al., 2012), rather than a reductionist variable-by-variable approach. In previous work (Stephens et al., 2020), we considered how aging was an important driver of metabolic change across a wide variety of metabolic biomarkers (anthropometric, fasting blood test and vital signs measurements), considering each one individually and noting a substantial degree of heterogeneity as to the impact of aging across them. In contrast, in the present study, we have used Complex Inference Networks (Stephens et al., 2009, 2018) of these biomarkers as a means to give a more holistic, systemsbiology perspective in order to demonstrate how the changes in the coupling between regulated variables and those regulatory systems that try to maintain homeostasis lead to metabolic health changes over a lifetime. In particular, in this paper, we will use complex physiological networks to better understand these interactions, constructing a data-driven network of biomarkers that can be used to characterize homeostasis and how it changes as a function of age.

\section{RESULTS}

\section{Demographic Description of the Population}

A general description of our study population $(n=2572)$, and the distinct age groups is provided in Table 1 . The mean age of the participants was 38 years old with a standard deviation, $S D=15$, and a range from 18 to 81 years old. Our population sample was predominantly female $(65 \%)$. This predominance 
TABLE 1 | Demographic description of the population.

\begin{tabular}{|c|c|c|c|c|c|c|c|}
\hline \multirow[b]{2}{*}{ Age range (min-max years) } & \multirow{2}{*}{$\begin{array}{l}\text { Total } \\
\text { 18-81 }\end{array}$} & \multicolumn{6}{|c|}{ Age groups } \\
\hline & & $<25$ & $25-34$ & $35-44$ & $45-54$ & $55-64$ & $>65$ \\
\hline Age (years \pm SD) & $38 \pm 15$ & $20 \pm 2$ & $30 \pm 3$ & $40 \pm 3$ & $50 \pm 3$ & $59 \pm 3$ & $70 \pm 4$ \\
\hline Sex (female \%) & $65 \%$ & $68 \%$ & $57 \%$ & $66 \%$ & $72 \%$ & $60 \%$ & $64 \%$ \\
\hline $\mathrm{n}$ & 2572 & 680 & 528 & 445 & 468 & 352 & 99 \\
\hline Basic education ${ }^{\star \star \star}$ & $16 \%$ & $2 \%$ & $6 \%$ & $13 \%$ & $20 \%$ & $21 \%$ & $22 \%$ \\
\hline Undergraduate $e^{\star \star \star}$ & $50 \%$ & $97 \%$ & $64 \%$ & $51 \%$ & $55 \%$ & $45 \%$ & $29 \%$ \\
\hline Postgraduate & $34 \%$ & $0 \%$ & $31 \%$ & $35 \%$ & $25 \%$ & $35 \%$ & $48 \%$ \\
\hline MetS prevalence $e^{\star \star \star}$ & $25 \%$ & $4 \%$ & $19 \%$ & $35 \%$ & $42 \%$ & $47 \%$ & $43 \%$ \\
\hline
\end{tabular}

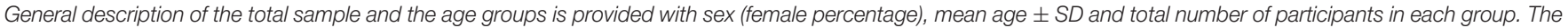
presence of a trend with age by chi-squared tests for trends is indicated by *** for $p<0.001$.

was preserved across age groups considered with no statistically significant differences between groups. Our population sample comes mainly from the metropolitan region of Mexico City (93\%), with the remaining participants from neighboring states. Educational level proportions changed within the age groups, with an increasing trend for postgraduate and basic education (at most 12 years of study), and a decreasing trend for undergraduate education, that are illustrative of the population composition within the sample (Table 1). We found that MetS prevalence, as defined by the harmonized criteria (Alberti et al., 2009), increased significantly by age (under a chi-squared test for trend $p<0.001)$, beginning with a prevalence of $4 \%$ for the first age group ( $<25$ years old), which increased ten-fold to $47 \%$ in the age group from 55 to 65 years old. For adults older than 65 years old, MetS prevalence is high (43\%) but is lower than that from 55 to 65 , however, this difference between groups is not statistically significant $\left[X^{2}(1, N=659)=0.14, p=0.7\right]$.

\section{Physiological Variables and Pathological State Prevalence Change With Age}

To examine whether this increase in MetS prevalence with age was due to an increment in the mean values of the physiological variables or to an increase in the tail of the distribution above the cut-off values (Table 2), linear regressions and chisquared tests for trends were evaluated (Table 3). Most of the physiological variables (fasting glucose, HbA1c, LDL cholesterol, triglycerides, urea, creatinine, waist, weight, systolic, and diastolic blood pressure) increased progressively with age, having a statistically significant positive linear regression slope, whereas height and axillar temperature decreased, being associated with a statistically significant negative linear regression slope. In contrast, three physiological variables: basal insulin, HDL cholesterol, and uric acid, showed no linear changes as a function of age. Following the trend of their respective physiological variables, the prevalence of pathological states also grew with age, with one exception: high temperature. While changes in the mean values of the physiological variables as a function of age were considerably smaller, as shown by the slopes in the linear regressions, the proportion of the population above the cut-off values increased substantially (Table 3). For the physiological variables, waist circumference, weight, systolic and diastolic pressure had the greatest regression coefficients as a function of age. Regarding the prevalence of pathological states, overweight, low estimated glomerular filtration rate (eGFR), and hyperglycemia, had the greatest increase as a function of age, followed by high blood pressure, high LDL, hypertriglyceridemia, high HbAlc, and azotemia. Age had a widespread influence on most of the components of MetS, whether regarded as continuous or as categorical variables. The prevalence of low HDL and hyperuricemia changed with age, although this trend was not detected by a linear regression.

\section{Metabolic Modules Can Be Identified Within the Network}

To investigate how metabolic physiological components are grouped within the networks, we employed two strategies, either identifying largest cliques (a clique is a group of fully connected nodes) or finding clusters within the networks (see Figure 1). For the first strategy, the largest cliques method shows the biggest possible, maximally connected subgraphs of a network, indicating which components go hand in hand most frequently across distinct age groups (Figures 1C,D). For the physiological network, weight, waist circumference, uric acid, systolic and diastolic blood pressures appeared most frequently in the major cliques (Figure 1C). In the pathological states network, insulin resistance, hypertriglyceridemia, overweight and hyperglycemia were most frequently found to occur within the largest cliques (Figure 1D). For the second strategy, the networks were assorted into different clusters, using the Louvain algorithm (Blondel et al., 2008) for the physiological network, or the Spinglass algorithm (Reichardt and Bornholdt, 2006) for the pathological states network (Figures 1E,F). Four main clusters were found in the physiological network (Figure 1E), with the main cluster associated with weight, and followed by a cluster around urea. An intermediary cluster was found around glucose and $\mathrm{HbA1c}$, while systolic and diastolic blood pressure remained separated from the rest. For the pathological states network, the main cluster was around hyperglycemia and the second was around low eGFR, with an intermediate cluster around high blood pressure and high temperature (Figure 1F). The metabolic components within these clusters were related by metabolic pathways, establishing metabolic modules. 
TABLE 2 | Pathological states criteria.

\begin{tabular}{|c|c|c|c|c|c|c|c|c|}
\hline & Physiological variables & & Pathological states & Cut-off value & Sex & Age & Organization & References \\
\hline 1 & Fasting glucose (mmol/L) & 1 & Hyper-glycemia & $>5.55 \mathrm{mmol} / \mathrm{L}$ & & & IDF & Alberti et al., 2009 \\
\hline 2 & $\mathrm{HbA1c}(\%)$ & 2 & High HbA1c & $>6.5 \%$ & & & ADA & $\begin{array}{l}\text { American Diabetes } \\
\text { Association, } 2020\end{array}$ \\
\hline 3 & Basal insulin (pmol/L) & 3 & Insulin resistance & $M>1.7 F>1.8$ & $\mathbf{x}$ & & - & $\begin{array}{l}\text { Esteghamati et al., } \\
2009\end{array}$ \\
\hline 4 & $\mathrm{HDL}(\mathrm{mmol} / \mathrm{L})$ & 4 & Low HDL & $\mathrm{M}<1.03 \mathrm{mmol} / \mathrm{L} F<1.3 \mathrm{mmol} / \mathrm{L}$ & $\mathbf{x}$ & & IDF & Alberti et al., 2009 \\
\hline 5 & $\mathrm{LDL}(\mathrm{mmol} / \mathrm{L})$ & 5 & High LDL & $>3 \mathrm{mmol} / \mathrm{L}$ & & & ESC/EAS & Mach et al., 2019 \\
\hline 6 & Triglycerides (mmol/L) & 6 & Hyper-triglyceridemia & $>1.7 \mathrm{mmol} / \mathrm{L}$ & & & IDF & Alberti et al., 2009 \\
\hline 7 & Uric Acid (umol/L) & 7 & Hyper-uricemia & $>405 \mathrm{umol} / \mathrm{L}$ & & & $\mathrm{ACR}$ & Khanna et al., 2012 \\
\hline 8 & Urea (mmol/L) & 8 & Azotemia & $>7.5 \mathrm{mmol} / \mathrm{L}$ & & & - & $\begin{array}{l}\text { Tyagi and Aeddula, } \\
2019\end{array}$ \\
\hline 9 & Creatinine (umol/L) & 9 & Low eGFR & $<90 \mathrm{ml} / \mathrm{min}$ & $\mathbf{x}$ & $\mathbf{x}$ & KDIGO & Levin et al., 2013 \\
\hline 10 & Waist (cm) & 10 & Overweight & $\mathrm{M}>90 \mathrm{~cm} \mathrm{~F}>80 \mathrm{~cm}$ & $\mathbf{x}$ & & IDF & Alberti et al., 2009 \\
\hline 11 & Weight (Kg) & & & & & & & \\
\hline 12 & Height (cm) & & & & & & & \\
\hline 13 & Axilar temperature $\left({ }^{\circ} \mathrm{C}\right)$ & 11 & High Temperature & $>37^{\circ} \mathrm{C}$ & & & - & $\begin{array}{l}\text { Sund-Levander et al., } \\
2002\end{array}$ \\
\hline 14 & Systolic (mmHg) & 12 & High Blood Pressure & $>120 / 80 \mathrm{mmHg}$ & & & ACC/AHA & Whelton et al., 2018 \\
\hline 15 & Diastolic (mmHg) & & & & & & & \\
\hline
\end{tabular}

Threshold values employed for the classification of pathological states. Current criteria that are tailored for age and sex are indicated in the columns.

Both strategies lead to a selection of nodes that differs from current MetS criteria (Figures 1A,B). While waist and weight are frequently part of the largest clique of the network, they are often clustered separately from the metabolic components of triglycerides and glucose. Triglycerides, both as a physiological variable or as pathological state, are frequently part of the largest cliques and belong to the main cluster of the networks. Hyperglycemia, on the other hand, is part of the main cluster only in the pathological states network and is frequently part of largest cliques but is not part of the largest cliques nor of the main cluster as a physiological variable (glucose). Systolic and diastolic blood pressures are also frequently part of the largest cliques, but only as physiological variables and not as a pathological state. They belong mainly to the cluster of overweight as pathological states, but are in an independent cluster as physiological variables. Finally, HDL cholesterol as a physiological variable was seldom part of the largest cliques; however, it was part of the main cluster in the pathological states network.

\section{The Role of Metabolic Biomarkers Within the Network Across a Lifetime}

The relations between the physiological variables and pathological states within the networks change with age. We observed that obesity, whether as proxied by the weight and waist circumference physiological variables, or as the overweight pathological state, is the main influencer in the network. This role was measured by eigencentrality, a measure of the first and second order connections of a node, and remained stable across all age groups (Figures 2A,C). In contrast, physiological variables with characteristically tight homeostatic control, like glycemic variables and temperature, were uninfluential in the network (Figure 2C). For the pathological states network, the largest influence, as measured by the hub score, a generalization of eigencentrality for directed graphs, where only outgoing links are measured, was exerted by overweight, with the components of dyslipidemia becoming less influential from 25 to 34 years old onward, while the pathological states associated with low estimated glomerular filtration rate (low eGFR) steadily became more relevant above 65 years old (Figures 2B,D). In order to assess which nodes are intermediaries in the network, a "betweenness" measure is required. The most useful here is betweenness flow, where flow is taken as the minimum weight associated with each disjoint path between any two nodes. The betweenness flow of a node is then the sum of the flows that are lost if that node is removed from the network. It is therefore a measure of how much flow is mediated by a given node. This property is called gatekeeping, since it represents the potential to disconnect the flow. High intermediacy biomarkers of the flow between systems were uric acid, insulin, HbAlc and HDL in the physiological network, while hypertriglyceridemia, insulin resistance, hyperglycemia and high $\mathrm{HbAlc}$ were the main intermediaries between pathological states (Figures 2E,F). While eigencentrality values are stable for each node regardless of age, flow betweenness values change profoundly as a function of age (Figures 2E,F).

\section{Whole Network Topology as a Biomarker for Metabolic Homeostasis}

As well as local properties of the physiological variables and pathological states networks, global properties also change with age. Topological properties of these networks for all the age groups are summarized in Table 4 . These topological properties describe the structure of the network in several aspects. For undirected networks transitivity and clustering coefficient, measures of the proportion of triangles in the network, and characteristic path length, a measure of the 
TABLE 3 | Physiological variables means and pathological states prevalence.

\begin{tabular}{|c|c|c|c|c|c|c|c|c|}
\hline & & \multicolumn{7}{|c|}{ Age groups } \\
\hline & & Total & $<25$ & $25-34$ & $35-44$ & $45-54$ & $55-64$ & $>65$ \\
\hline Physiological variable & Slope & \multicolumn{7}{|c|}{ Mean \pm SD } \\
\hline Waist (cm) & $0.3^{\star \star \star}$ & $88 \pm 12$ & $82 \pm 11$ & $87 \pm 12$ & $91 \pm 12$ & $93 \pm 12$ & $93 \pm 11$ & $94 \pm 12$ \\
\hline Systolic blood pressure (mmHg) & $0.3^{\star \star \star}$ & $113 \pm 14$ & $109 \pm 11$ & $109 \pm 12$ & $113 \pm 14$ & $115 \pm 14$ & $121 \pm 16$ & $124 \pm 19$ \\
\hline Diastolic blood pressure (mmHg) & $0.2^{\star \star \star}$ & $74 \pm 10$ & $71 \pm 9$ & $73 \pm 9$ & $76 \pm 11$ & $77 \pm 10$ & $79 \pm 11$ & $78 \pm 11$ \\
\hline Weight (Kg) & $0.17^{\star \star \star}$ & $68 \pm 15$ & 63. \pm 13 & $68 \pm 15$ & $72 \pm 15$ & $71 \pm 14$ & $70 \pm 14$ & $68 \pm 13$ \\
\hline Basal insulin (pmol/L) & $0.12 \mathrm{~ns}$ & $58 \pm 48$ & $55 \pm 36$ & $54 \pm 52$ & $57 \pm 42$ & $60 \pm 62$ & $59 \pm 40$ & $59 \pm 44$ \\
\hline Creatinine (umol/L) & $0.12^{\star \star}$ & $72 \pm 29$ & $70 \pm 14$ & $74 \pm 45$ & $70 \pm 16$ & $70 \pm 17$ & $74 \pm 18$ & $88 \pm 78$ \\
\hline Uric Acid (umol/L) & $0.09 n s$ & $322 \pm 87$ & $323 \pm 92$ & $326 \pm 90$ & $319 \pm 87$ & $315 \pm 82$ & $331 \pm 88$ & $335 \pm 85$ \\
\hline Fasting glucose (mmol/L) & $0.028^{\star \star \star}$ & $5 \pm 1.5$ & $4.6 \pm 0.5$ & $4.7 \pm 0.7$ & $5 \pm 1$ & $5 \pm 2$ & $5 \pm 2$ & $6 \pm 2$ \\
\hline Urea (mmol/L) & $0.026^{\star \star \star}$ & $5 \pm 1$ & $4 \pm 1$ & $5 \pm 2$ & $4 \pm 1$ & $5 \pm 1$ & $5 \pm 1$ & $6 \pm 2$ \\
\hline HbA1c (\%) & $0.022^{\star \star \star}$ & $5.5 \pm 1$ & $5.1 \pm 0.5$ & $5.1 \pm 0.6$ & $5 \pm 2$ & $6 \pm 2$ & $6 \pm 2$ & $6 \pm 2$ \\
\hline Triglycerides (mmol/L) & $0.019^{\star \star \star}$ & $1.6 \pm 1$ & $1.1 \pm 0.6$ & $1 \pm 1$ & $2 \pm 2$ & $2 \pm 1$ & $2 \pm 1$ & $2 \pm 1$ \\
\hline LDL (mmol/L) & $0.016^{\star \star \star}$ & $3 \pm 1$ & $2.5 \pm 0.6$ & $3 \pm 1$ & $3 \pm 2$ & $3.2 \pm 0.8$ & $3.3 \pm 0.9$ & $3.2 \pm 0.8$ \\
\hline $\mathrm{HDL}$ (mmol/L) & $0.0004 n s$ & $1.2 \pm 0.3$ & $1.3 \pm 0.3$ & $1.2 \pm 0.3$ & $1.2 \pm 0.3$ & $1.2 \pm 0.3$ & $1.2 \pm 0.3$ & $1.3 \pm 0.3$ \\
\hline Height (cm) & $-0.098^{\star \star \star}$ & $162 \pm 9$ & $162 \pm 9$ & $164 \pm 9$ & $162 \pm 9$ & $159 \pm 9$ & $160 \pm 10$ & $159 \pm 10$ \\
\hline Axilar temperature $\left({ }^{\circ} \mathrm{C}\right)$ & $-0.0053^{\star \star \star}$ & $37 \pm 0.5$ & $37.2 \pm 0.5$ & $37.0 \pm 0.5$ & $37.0 \pm 0.5$ & $37.0 \pm 0.5$ & $36.8 \pm 0.5$ & $36.7 \pm 0.6$ \\
\hline Pathological states & Chi-square & \multicolumn{7}{|c|}{ Prevalence (\%) } \\
\hline Overweight & 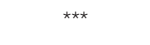 & 60 & 38 & 52 & 73 & 80 & 82 & 80 \\
\hline Low eGFR & $463^{\star \star \star}$ & 22 & 4.6 & 11 & 18 & 34 & 51 & 75 \\
\hline Hyperglycemia & $228^{\star \star \star}$ & 13 & 1.5 & 4.4 & 12 & 23 & 28 & 31 \\
\hline High Blood Pressure & $202^{\star \star \star}$ & 21 & 11 & 11 & 22 & 28 & 42 & 41 \\
\hline High LDL & $189^{\star \star \star}$ & 49 & 24 & 42 & 51 & 62 & 65 & 62 \\
\hline Hyper-triglyceridemia & $159^{\star \star \star}$ & 33 & 14 & 32 & 41 & 44 & 48 & 43 \\
\hline High $\mathrm{HbA} 1 \mathrm{c}$ & $116^{\star \star \star}$ & 5.6 & 0.7 & 0.6 & 5.2 & 8.8 & 12 & 18 \\
\hline Azotemia & $41^{\star \star \star}$ & 3.2 & 0.4 & 2.4 & 3.8 & 6.1 & 7.5 & 15 \\
\hline Insulin resistance & $10.3^{\star \star}$ & 14 & 9.2 & 12 & 13 & 14 & 16 & 12 \\
\hline Low HDL & $8.8^{\star \star}$ & 47 & 43 & 46 & 53 & 50 & 42 & 39 \\
\hline Hyperuricemia & $4.5^{\star}$ & 15 & 13 & 19 & 14 & 14 & 18 & 21 \\
\hline High Temperature & $0.05 n s$ & 1.1 & 0.9 & 0.4 & 0.9 & 1.7 & 0.6 & 0 \\
\hline
\end{tabular}

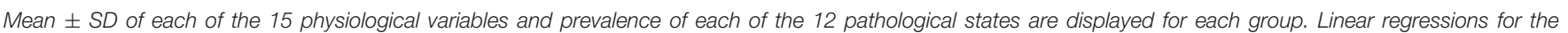

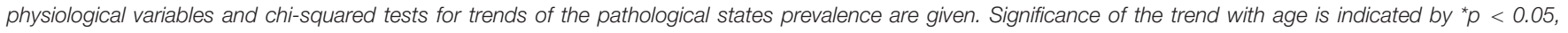
${ }^{* *} p<0.01$, and ${ }^{* *} p<0.001$. ns, not statistically significant.

distance between nodes, are important descriptors of structure. For directed networks a third parameter is reciprocity, the proportion of bilateral connections in the network. Noticeably, for the pathological states network, we found that reciprocity was lower and transitivity was greater than would be expected for random networks of the same size, number of links or dyads (Table 4). Characteristic path length was lower than would be expected for random networks. Moreover, the local transitivity of physiological variables reaches a peak in the life decade between 25 and 34 years old, and from then on, the transitivity begins to decrease (Figures 3A,C). However, this decrease is not the result of a reduction in the weighted degree distribution (strength) of the correlations within the network, which are similar across all age groups (Figure 3E), instead it is related to an increase in the number of edges within the network, as presented by network density (Table 4). In other words, the organization of the physiological variables changed independently from the strength of the relationships between the variables. Over a lifetime, nodes within a cluster tend to connect more within themselves rather than outside the cluster. This topological change results in a modularity increase in the physiological network (Figure 3D). However, this trend was not shared with the pathological states network. In this network, there is a trend toward increasing transitivity until the 45 to 54 years old age groups group, and a decrease in older groups (Figures 3B,C). Pathological states became increasingly correlated as a function of age, until reaching a maximum in the decade between 45 and 54 years old (Figure 3C). This clustering change is related to the weighted degree distribution of the pathological states network (Figure 3F) and to an increase in the density of the network (Table 4). In these networks modularity, a measure of how well separated are the clusters, decreases from the 35 to 44 years old group onward (Figure 3D). Three stages become apparent: a healthy stage, where the clustering of both networks increases; a transition 
A

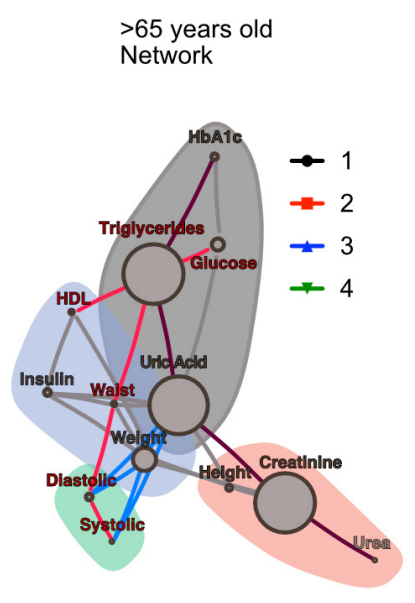

C

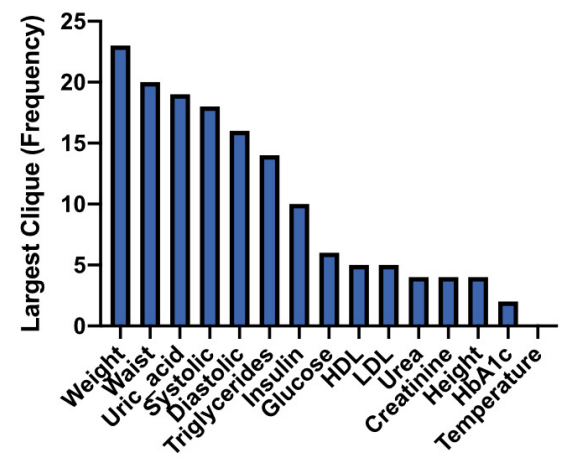

E

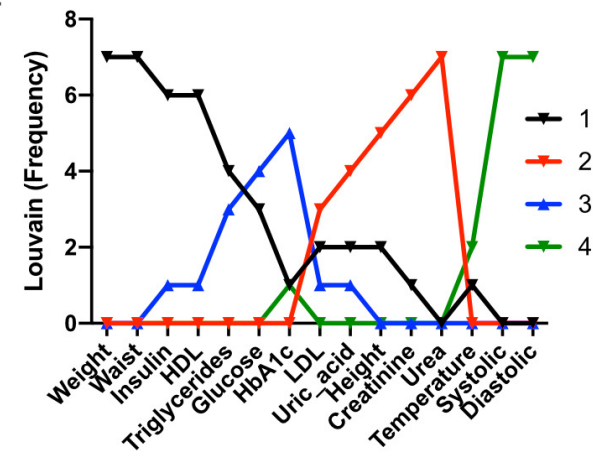

B

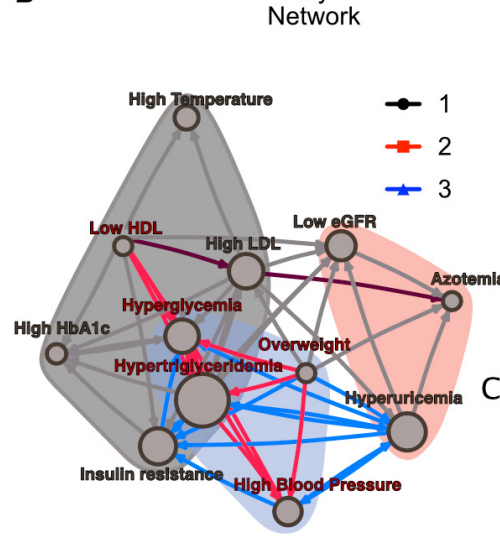

Metabolic

syndrome

Largest clique

Farthest nodes

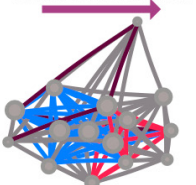

Cliques \& subgraphs

D

Pathological network
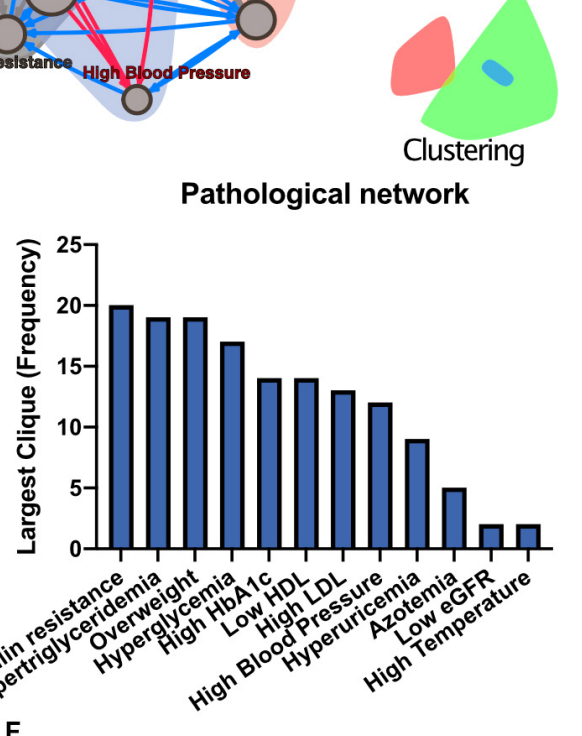

$F$

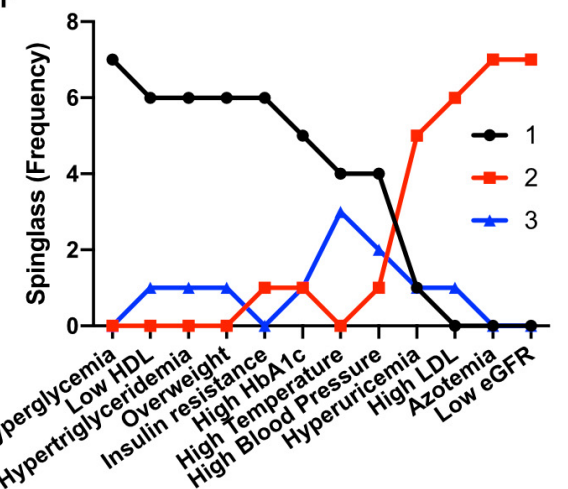

FIGURE 1 | Physiological subsystems identified by Data-driven association. Representative networks (A) for the physiological variables network and (B) for the pathological states network. Physiological variables and pathological states clusters are shown as largest cliques (blue connections), and, as clusters (nodes within color highlighted areas). In both metabolic physiological networks, the red subgraph shows the currently accepted MetS components. The diameter of the network the two furthest nodes path - is highlighted in purple. (C) Frequency of physiological variables composing the largest clique of each age group network.

(D) Frequency of pathological states fully associated within largest cliques as shown by the pathological states network. The frequency of appearance of a node pertaining to a certain cluster (membership) was registered. Since 7 networks were generated (all participants, and 6 age-range groups), a node belonging to the same cluster across the entire lifespan would reach a value of 7 . In panel (E), the frequency value represents how many times a node is part of the same cluster for the physiological variables, where the Louvain algorithm was used to determine clusters. Three main clusters appear, with blood pressure variables making a fourth. (F) Cluster membership of pathological states using the spinglass community algorithm that selects the group of nodes most likely to be found in the same state.

Three main clusters appear, with different groups of pathological states in each one.

stage, where the clustering of pathological states increases, while the clustering of physiological variable decreases; and a disease stage, where the clustering of both networks decreases
(Figure 3C). The proportion between clustering coefficient and characteristic path length in a network can be summarized by the small world index to compare structural changes in 

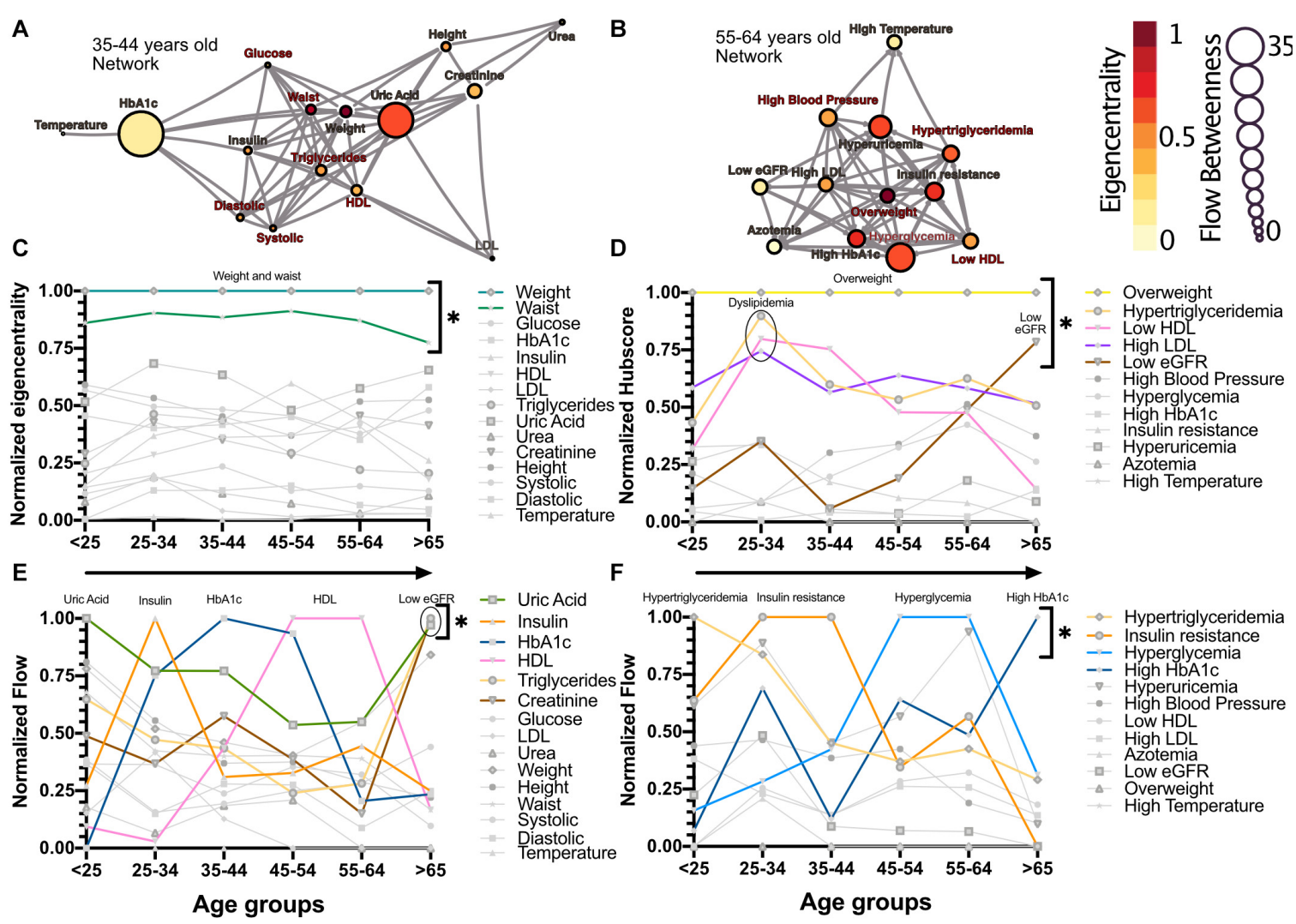

FIGURE 2 | Network modeling highlights physiological and pathological interactions. Centrality measurements identify the role of each physiological variable or pathological state within the metabolic network. (A) Physiological network from 35 to 44 years old, and (B) pathological network from 55 to 64 years old, as examples of the different centrality contribution that each node has. Influence is measured by eigencentrality and is represented by node color, while betweenness is measured by flow and represented by node size. The values from these examples are emphasized inside gray rectangles. (C) Most influential nodes in the physiological variables network, Weight and waist, are indicated. (D) Most influential nodes as seen by eigencentrality in the pathological states network. Overweight, dyslipidemia and low eGFR are indicated. (E) Gatekeeping nodes, as seen by flow betweenness, that mediate the associations between those physiological variables that are not directly connected. (F) Gatekeeping nodes that are the route between unconnected pathological states. The most meaningful nodes in this regard are hypertriglyceridemia, insulin resistance, hyperglycemia and high $\mathrm{HbA1C}$ as age increases. * indicates values unlikely to be found by chance alone in CUG tests.

TABLE 4 | Topological properties of the physiological variables and pathological states networks.

Total

Age groups

\begin{tabular}{llllll}
\hline$<25$ & $25-34$ & $35-44$ & $45-54$ & $55-64$ & $>65$
\end{tabular}

Physiological variables networks

Density

Global transitivity

$\begin{array}{ll}0.73 & 0.47\end{array}$

0.79

0.71

0.63

0.53

0.74

0.70

1.40

1.57

1.38
0.67

0.78

0.69

1.3

1.4

0.40

0.40

0.04

0.06

0.07

0.33

0.79

0.79

1.19

0.69

1.15

1.23

1.27
0.41

0.41

0.43

3.5

3.3

3.1

0.49

0.39

0.26

0.70

0.67

0.47

1.67

1.54

1.85

0.50
2.8

3.5

0.63

0.58

0.51

1.5

2.1

2.3

Smallworld Index

2.8

0.42

0.39

0.30

0.04

0.03

Global measurements for topological properties are shown for each network. In the case of the pathological states network, since it is directed, reciprocity of the network is also shown. 


\section{A}

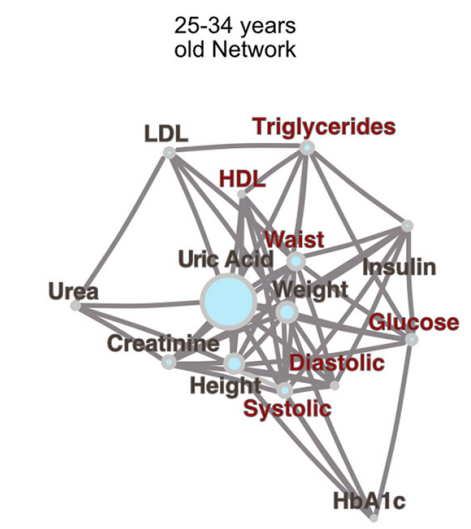

C

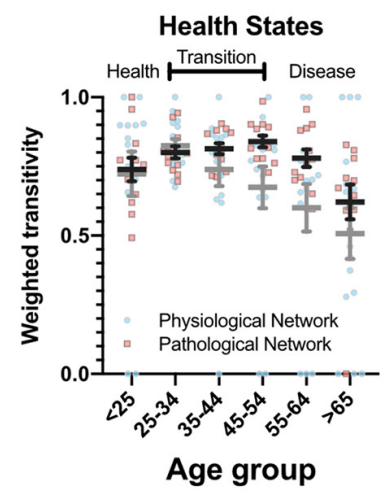

E

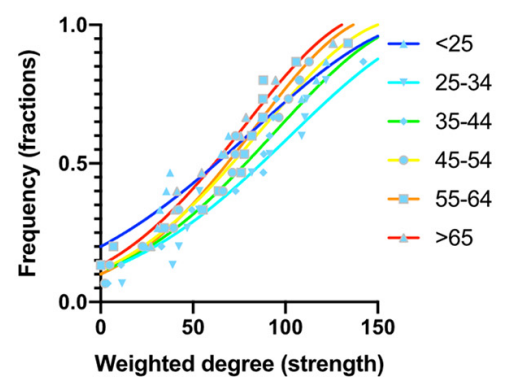

B 45-54 years

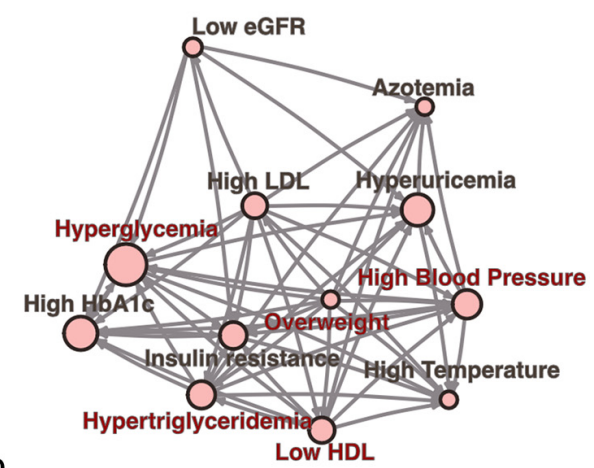

D

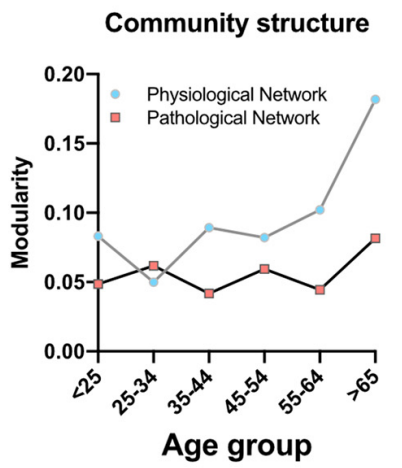

$\mathbf{F}$

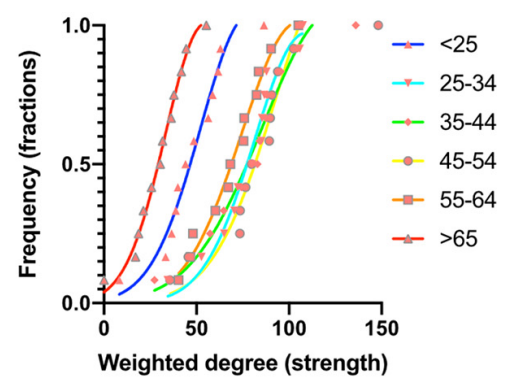

FIGURE 3 | Topological properties from physiological and pathological networks. Network structural changes as a function of age can be seen using several topological metrics. (A) Physiological network of the third decade of life as a visual example of weighted transitivity in a tightly intertwined network. (B) Pathological network of the fifth decade of life as an example of weighted transitivity in a directed network. These two networks represent the greatest transitivity in all age groups. (C) Weighted transitivity of each network as the mean \pm S.E.M. from all life decades, $n=2572$. The values that come from the physiological network nodes are highlighted in blue and for the pathological states network in pink. (D) Weighted transitivity of each network as the mean \pm S.E.M. value of the 12 tested pathological states from all the age groups. Frequency distribution of the weighted degree (strength) of the network in each life decade (E) for the physiological networks and (F) for the pathological states networks. Age dissociates physiological variables, as seen by the reduction of the weighted transitivity in the physiological network, but without a significant change in the weighted degree, while pathological conditions become more associated with age, as seen in the pathological network, reaching a peak at the fifth decade of life.

our matching networks of increasing age. For the physiological networks of groups starting below 54 years, the small-world index has values between 1.3 and 1.9 , increasing to values above 2 in the groups above 55 years old. All pathological networks had a greater small world index than the corresponding physiological networks, which increased substantially in the age group above 65 years old and concurrently with a decrease in the global clustering coefficient.

\section{DISCUSSION}

Metabolic homeostasis loss is the main driver of noncommunicable diseases and their resulting mortality. These complex diseases involve diverse combinations of risk biomarkers that occur more often together than by chance alone (Alberti et al., 2009). Currently, however, only five such factors are monitored for the assessment of metabolic health 
(overweight, high triglycerides, low HDL cholesterol, high systolic blood pressure, and high fasting plasma glucose). By adopting a network approach, in this study, we have shown that, in reality, not only the level of each individual factor is important, but also their correlations, both local and global. Local properties of the network are equivalent to current reductionist approaches, while global properties provide new metrics that can be used as markers of metabolic health. As allostatic load on body metabolism increases with age, changes in the ratios between different physiological variables represent the adaptive adjustment of their corresponding setpoints in order to accommodate an increasing burden of internal failures and cumulative external insults (Fossion et al., 2018; Goldstein, 2019). Here, we have shown that the number of correlations present within the networks, represented as network density (Table 4), the number of connections of each node, represented as the node's degree, and the strength of the correlation, represented as weighted degree, all change gradually across age groups and reflect this adaptive adjustment (Figure 3). Therefore, topological properties that emerge from the structure of the networks reflect how whole-system interactions within the physiological network change over a lifetime and, in particular, show how, as age increases, the loss of metabolic homeostasis is revealed by these changes. For example, local weighted transitivity measures the probability that the neighbors of a node are connected among themselves. This measure has the advantage of being largely independent from the size of the network (Barabási et al., 2003). Changes in this metric give insight into how the cumulative impact of metabolic insults increases and decreases the relations between physiological variables and pathological states. At the global level, transitivity and the clustering coefficient of the network are two indicators of how the network's connections become aggregated or disaggregated as a function of age. Therefore, these changes in the networks' structure echo the underlying homeostatic changes.

The transition from health to disease, in the case of complex diseases, can be described by three-state models (Chen et al., 2017). In the healthy stage, regulated variables are kept within strict bounds and physiological response systems increase their activity proportionally in order to compensate the impact of interaction with the environment. In the transition stage (from 35 to 54 years old) regulated variables increase their correlation with their physiological response system as metabolic insults are not fully compensated. At this stage internal malfunctions can be buffered, but at the expense of the development of pathological states, that then begin to correlate, leading to an ever-increasing burden (Figure 3). Finally, homeostasis is lost, and pathological states lead to disease onset in an irreversible fashion, resulting in a decrease in the clustering of both network types. Regulated variables are now fully dysregulated from their corresponding regulatory system variables and correlations are lost. Our results show that the transition from health to disease is reflected in our topological metrics as a result of the changes in the correlations between physiological variables and the corresponding association between pathological states. The different network metrics we evaluated show that our networks are not random (Table 4). Although a formal, largescale topological characterization of our physiological networks falls beyond the scope of this work, and would potentially require the addition of many more variables, it is interesting to point out that the observed properties of scale free and small world are properties that are frequently found in complex biological systems (Song et al., 2005). It has been argued that these topologies confer properties of network robustness and adaptability that are desirable as properties with a homeostatic interpretation (Fossion et al., 2018; Toledo-Roy et al., 2019). Nevertheless, considering the wide structural diversity found in real-world networks, classification of these complex systems remains an active area of development (Hilgetag and Goulas, 2016; Broido and Clauset, 2019).

Individual biomarkers were described in the context of the network through centrality measurements of influence and intermediacy. The important influence of weight on the metabolic network was found in both network approaches and was sustained across all age groups (Figure 2). Additionally, weight-associated physiological variables and their corresponding pathological states were most frequently embedded within the largest cliques. Both these results exhibit the central role of weight inside the metabolic networks. This has been confirmed in a large cross-sectional study, where long term sustained weight loss was seen to improve overall metabolic risk (Knell et al., 2018). However, some classically established MetS components, such as HDL cholesterol, are seldom present within the largest cliques, indicating a more peripheral role within this network. In this regard, some of the biomarkers we used have a high flow betweenness in the network, suggesting that they behave as an "exchange currency" among several metabolic subsystems. This was the case for triglycerides, insulin, uric acid and glucose, whether considered as physiological parameters or as pathological states (Figure 2). These nodes have the potential of disconnecting the flow within the network, and therefore may serve as sensitive indicators of alterations from several different systems. This suggests that they are key components in the transmission of disruptions between different metabolic subsystems. Additionally, these metabolic subsystems, as identified by our clustering strategies, are also those that would be considered as the natural ones from a medical perspective (Goh et al., 2007; Chan and Loscalzo, 2012). Our results show that different, relatively independent, metabolic modules arise, that communicate through some gatekeeping exchange molecules. With age, this modularity increases in the case of the physiological variables network (Figure 3C). Such modularity is a measure of how much the networks tend toward a community structure. Furthermore, there is a strong correspondence between the clusters that were found in the physiological variables network and those found in the pathological states network, suggesting that the associated pathological states emerged from the underlying relationships between the corresponding physiological variables and are, therefore, not just a byproduct of chance or prevalence alone. These two approaches complement each other, reinforcing their respective conclusions where both reach similar results. This was the case for the clustering of metabolic components 
in both the physiological variables networks, the pathological states networks (Figures 1E,F), and the corresponding centrality measurements (Figure 2).

Finally, it is worth mentioning that another advantage of network analysis is that it can be used as part of an automated process for discovering and analyzing patterns in large datasets, with the assistance of experts to ensure a relevant and adequate interpretation (Merico et al., 2009). In this way, networks can be extended in an iterative process in order to accommodate new biomarkers in a way that can both enrich and refine the

A

Network construction

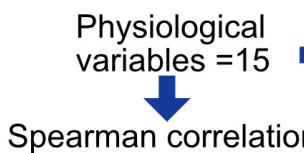

B

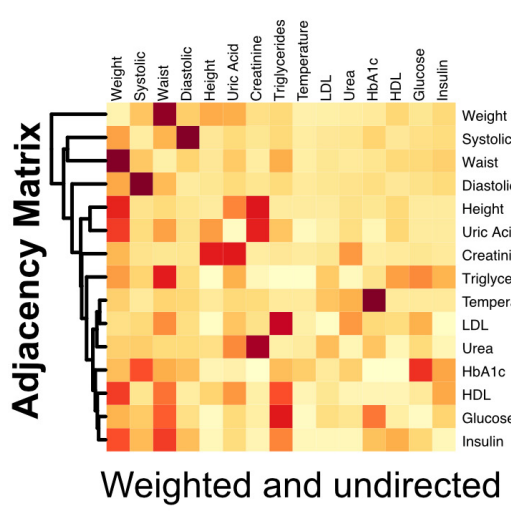

D

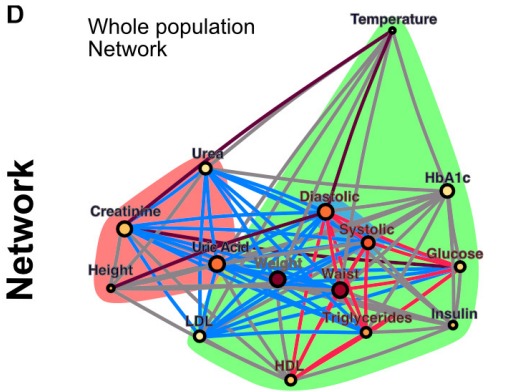

F Physiological network
1) $\mathrm{T}$

Degree and clustering coefficient

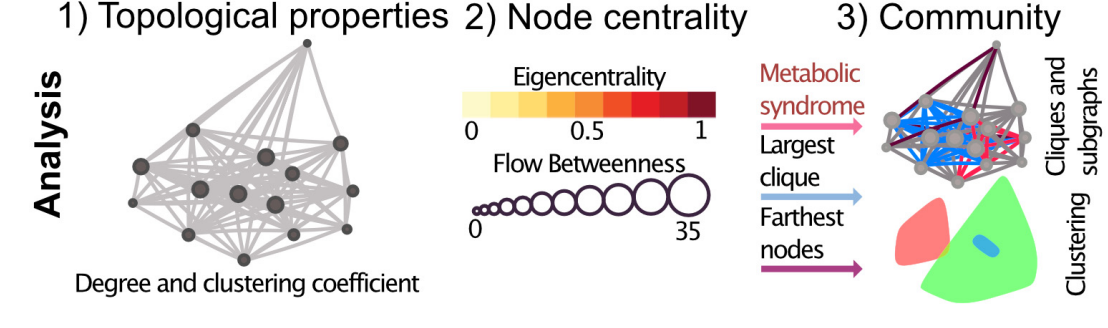

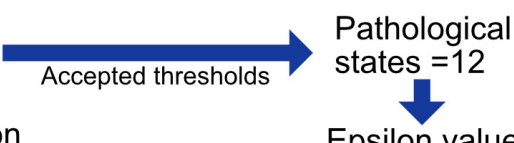

C Epsilon value

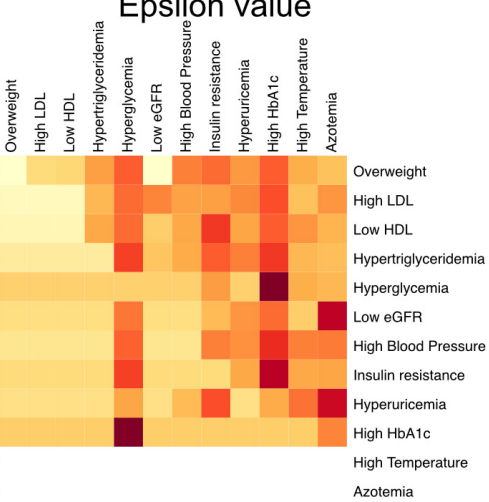

Weighted and directed

E

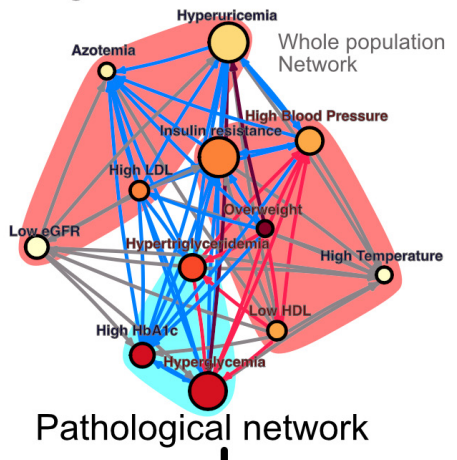

Pathological network

2) Node centrality

3) Community

FIGURE 4 | Metabolic physiological network construction from matrices. (A) Correlation of 15 physiological variables and their corresponding 12 pathological states associations were modeled using Spearman correlation and $\varepsilon$ value, respectively. (B) Adjacency matrix as a heatmap where the darker the red indicates a greater monotonic relationship between two physiological variables, as calculated by the Spearman rank correlation rho. (C) $\varepsilon$ Value between each pair of pathological states, a darker red indicating a greater probability of coexistence. In both heatmaps, rows and columns are ordered by weighted degree, and on the left side of the heat maps the resulting hierarchical dendrogram is shown. For directed networks some nodes lacked outgoing links, this is presented as blank rows. (D) Undirected network of physiological variables for the whole sample. The edges are weighted by the rho value in the Spearman correlation. The size of the node shows the flow betweenness of a node, the eigencentrality is shown by its color and the color shadowed areas indicate the Louvain clusters. (E) Directed network of pathological states. The edges are weighted by the $\varepsilon$ value, the size of the node shows the flow betweenness of each node, the eigencentrality is shown by its color and the color shadowed areas indicate spinglass clusters. (F) In both networks, the red subgraph shows the components of MetS, while the blue subgraph highlights the largest clique and the diameter of the network is in purple. For Spearman correlation, values with $p>0.001$ were discarded, whereas for $\varepsilon$, values below 1.96 were discarded. 
TABLE 5 | Glossary.

\begin{tabular}{|c|c|c|c|c|}
\hline Glossary & Symbol & Definition & R_package:function & References \\
\hline Adjacency matrix & A & $\begin{array}{l}\text { An array of rows and columns that contains the } \\
\text { connections of the network }\end{array}$ & & \\
\hline Subgraph & $S \subseteq V$ & $\begin{array}{l}\text { A subset of nodes and their links contained in the original } \\
\text { network }\end{array}$ & igraph:induced.subgraph & \\
\hline Vertex & V & A node & & \\
\hline Degree & & Number of links that a node has & sna:degree & Freeman, 1979 \\
\hline Strength & & The sum of the weights of the links attached to a node & igraph:strength & Barrat et al., 2004 \\
\hline Flow & $f$ & $\begin{array}{l}\text { A measure that describes the strength of the links in a path } \\
\text { between nodes }\end{array}$ & & \\
\hline Hub score & $\begin{array}{l}\text { Eigencentrality } \\
\text { from } A^{*}(A)\end{array}$ & $\begin{array}{l}\text { Eigencentrality of the matrix that takes into account only } \\
\text { out-going links }\end{array}$ & igraph:hub_score & Kleinberg, 1998 \\
\hline Medial measures & & $\begin{array}{l}\text { Those centralities that are based on the number of walks } \\
\text { that pass through a node }\end{array}$ & & $\begin{array}{l}\text { Borgatti and } \\
\text { Everett, } 2006\end{array}$ \\
\hline Flow betweenness & & $\begin{array}{l}\text { The amount of flow mediated by a given node. This } \\
\text { illustrates the gate-keeping role of a node, i.e., the potential } \\
\text { to disconnect the network. }\end{array}$ & sna:flowbet & $\begin{array}{l}\text { Koschützki and } \\
\text { Schreiber, } 2008\end{array}$ \\
\hline Cluster, community & & $\begin{array}{l}\text { A set of nodes with many links between themselves and } \\
\text { few nodes to the outside of the community (the rest of the } \\
\text { network). }\end{array}$ & & Blondel et al., 2008 \\
\hline Clique & & $\begin{array}{l}\text { A subgraph where all nodes are fully connected between } \\
\text { themselves. }\end{array}$ & & $\begin{array}{l}\text { Eppstein et al., } \\
2010\end{array}$ \\
\hline Density & & $\begin{array}{l}\text { The ratio of links that are present in a network to all the } \\
\text { possible edges it could contain. }\end{array}$ & igraph:graph.density & $\begin{array}{l}\text { Faust and } \\
\text { Wasserman, } 1994\end{array}$ \\
\hline Reciprocity & & The ratio of bidirectional links in a directed graph. & igraph:reciprocity & \\
\hline Characteristic path length & $\mathrm{L}$ & $\begin{array}{l}\text { The average of all the shortest paths between each pair of } \\
\text { nodes in the network }\end{array}$ & igraph:average.path.length & West, 1996 \\
\hline Transitivity, local & & $\begin{array}{l}\text { Transitivity and clustering coefficient are two slightly different } \\
\text { ways of counting triangles in a network. Both can be local, } \\
\text { when only one node and their neighbors are considered, or } \\
\text { global, when the whole network is considered. It represents } \\
\text { the ratio of all the triangles present to all the possible } \\
\text { triangles in the network. }\end{array}$ & igraph:transitivity & Barrat et al., 2004 \\
\hline Transitivity, global & $\mathrm{T}$ & & igraph:transitivity & Barrat et al., 2004 \\
\hline Clustering coefficient, local & & & DirectedClustering:ClustF & $\begin{array}{l}\text { Onnela et al., 2005; } \\
\text { Fagiolo, } 2007\end{array}$ \\
\hline Clustering coefficient, global & $\mathrm{CC}$ & & DirectedClustering:ClustF & $\begin{array}{l}\text { Onnela et al., 2005; } \\
\text { Fagiolo, } 2007\end{array}$ \\
\hline Small world index & SWI & $\begin{array}{l}\text { A measure that describes the relation between } C C \text { and } L \text { in } \\
\text { a network against what would be expected in a random } \\
\text { network. }\end{array}$ & qgraph:smallworldlndex & $\begin{array}{l}\text { Watts and Strogatz, } \\
1998\end{array}$ \\
\hline
\end{tabular}

A brief description for quick reference of specialized terms. 
generated network models (Aittokallio and Schwikowski, 2006). Unfortunately, many of these biomarkers cannot be monitored continuously, or their measurement is relatively expensive. However, the correlation networks that arise from transversal studies that consider a wide age spectrum can provide a means for studying the relations among physiological variables at a population level, while at the same time reducing the costs and difficulties associated with a longitudinal study. Of course, there are subtleties and limitations associated with the interpretation of such transversal data that apply equally to our work. In spite of this, comparisons between cross-sectional and longitudinal data, as well as retrospective studies, are in good agreement with the trends presented in Table 3, which reinforce the role of aging as the origin of the changes we observe (Chiu et al., 2015; Gu et al., 2018). Transversal studies are complementary to longitudinal approaches and result in an useful approximation (Fossion et al., 2017). In fact, the narrow age cohort approach we have employed is useful for demonstrating the increasing (and decreasing) covariances that occur between variables due to the underlying aging process. Nevertheless, there is, of course, no cross-sectional design that can account for the correlated changes that occur within a given individual (Hofer and Sliwinski, 2001). In addition, although our study is transversal in nature, and the generated networks are static, certain network properties, such as a low characteristic path lengths and high clustering coefficient in small world networks, are known to affect dynamic properties, such as the velocity of the spread of a disease (Jansson, 2020).

Our work provides the layout for evidence-based rationale for adding (or replacing) other CVD risk factors (e.g., CRP or family history) to the definition of MetS (Kahn et al., 2005). For instance, the physiological variables network does not rely on the particular values of cut-offs and illustrates that some variables that are not monitored currently, such as uric acid, may be better early indicators of metabolic burden. It is important to notice that uric acid is not used traditionally as a biomarker of metabolic disorders, even when in our network analysis it is more frequently embedded within the largest cliques than blood pressure components, triglycerides and HDL cholesterol (Figure 1C). This result adds to the growing body of literature that considers uric acid to be a relevant biomarker in MetS (Kanbay et al., 2016). In summary, the physiological network approach to metabolic homeostasis is capable of providing useful insights on whole-system function that are inaccessible through reductionist approaches.

\section{CONCLUSION}

Changes in network topology are global indicators of metabolic homeostasis and do not rely on any single parameter or threshold but, instead, assess the behavior of the whole system. Thus, this novel conceptualization of homeostatic health allows for a more holistic comprehension of a person's physiology. Structural properties, such as weighted transitivity or the small-world index, may then serve as topological indicators of health for the metabolic physiological network.

\section{METHODOLOGY}

\section{Ethical and Human Research Considerations}

This study was carried out in accordance with current regulation contained in the Mexican Official Normativity, NOM-012-SSA32012. The Ethics Committee of the Facultad de Medicina of the UNAM approved the procedures and protocols for this study under project FM/DI/023/2014, all the participants provided a written informed consent.

\section{Study Population and Age Sub-Groups}

We performed a transversal, community-based study of an ethnically and educationally diverse sample within a large public university, comprising 2572 participants. Each participant answered a health questionnaire and underwent vital signs, and anthropometric measurements along with fasting blood tests. This resulted in a multi-dimensional data set. The sampling was performed in successive steps from 2014 to 2019. The global sample was divided into 6 groups of increasing age, beginning with less than 25 years, and increasing in decades up to above 65 years of age. As a result, we obtained 6 age groups (see Table 1).

\section{Anthropometric Measurements and Laboratory Procedures}

All tests were performed in the morning during a 4-h period (from 6 a.m. to 10 a.m.) after verifying fasting and general status. Anthropometric measurements (weight, height, waist and hip circumferences) and vital signs (blood pressure and temperature) were taken by trained medical staff using standard procedures (World Health Organization [WHO], 1995; Whelton et al., 2018). Blood samples were obtained from participants who had fasted for 8 to $12 \mathrm{~h}$. Samples were stored at $4-5^{\circ} \mathrm{C}$ and submitted for chemical analysis of glucose, glycated hemoglobin (HbAlc), insulin, triglycerides, total cholesterol, HDL cholesterol, LDL cholesterol, uric acid and creatinine. Fasting plasma glucose was measured using spectrophotometry and potentiometry with a hexokinase kit (amorting PIPES, NAD, Hexokinase, ATP, $\mathrm{Mg}^{2+}$, G6P-DH; AU 2700 Beckman Coulter R). HbA1c was measured with High Performance Liquid Chromatography (HPLC) analysis with the Variant R Turbo kit 2.0, which consisted of 2 buffers and 1 wash solution. Fasting plasma insulin concentrations were determined using Chemiluminescence (Access Ultrasensitive Insulin, Unicell Dxl 800 Beckman Coulter R, Sensitivity: 0.03-300 U/mL). The lipid profile was obtained with enzymatic colorimetric assay (glycerol phosphate oxidase, cholesterol oxidase, acceleratorselective, detergent, and liquid-selective detergent). Uric acid was measured using the colorimetric method with uricase enzymatic OSR6698, system AU2700/5400, Beckmann Coulter R. This resulted in a set of 15 non-derivative, independent, continuous, physiological variables. From the original data set, 14 particular values associated with distinct variables were excluded, based on two main criteria:

(1) Outliers based on physiologically improbable values that are most likely to be erroneous as they would be incompatible 
with life. This included removing three values of blood pressure, three values of axillar temperature, two glucose measurements, two values of $\mathrm{HbAlc}$, and one each of uric acid, and LDL.

(2) Anthropometric measurements which were inconsistent between themselves. For example, exceedingly high values of waist circumference in an underweight participant. Thirteen values of waist and one value of height were discarded on this account.

\section{Pathological States Assessment}

From these physiological variables, thresholds were defined in order to distinguish normal values from abnormal values, thus categorizing health status or a pathological state (Table 2). We would like to emphasize that the thresholds used here are not diagnostic of disease; instead they are low enough values that indicate increased risk. Most of our criteria are backed up by major health societies and organizations, however, when a consensus was not available, we used literature-based cut-off values that best correlated with the increased risk-prevention view of the harmonized MetS criteria (Sund-Levander et al., 2002; Alberti et al., 2009; Esteghamati et al., 2009; Khanna et al., 2012; Levin et al., 2013; Whelton et al., 2018; Mach et al., 2019; Tyagi and Aeddula, 2019; American Diabetes Association, 2020). Thus, the pathological states described here are not diseases per se, but an indication that physiological values do not represent normal health status. Three of the physiological variables that we measured do not have a pathological state by themselves alone. For instance, high blood pressure was determined by either elevated systolic or diastolic values. For insulin and creatinine, two derived indices were calculated: Homeostasis Model Assessment Insulin Resistance index (HOMA-IR) (Wallace et al., 2004) for the pathological state of insulin resistance, and eGFR for chronic kidney disease (Levin et al., 2013).

\section{Network Modeling}

Network science is now an important are of science in itself with applications in many different fields. The construction of complex networks of nodes, and links between them that represent interactions, permits the simultaneous visualization and analysis of potentially large numbers of such interactions where global properties of the system that are not apparent at the local level manifest themselves. The vast majority of networks have links that are associated with known, experimentally verified interactions, such as in a food web or a social network. In this paper, however, we will use Complex Inference Networks (Stephens et al., 2009, 2018), where the interaction represented by a network link is inferred rather than directly observed, by examining co-occurrences between variables. Such cooccurrences may be in space or time, or both. Here, we consider co-occurrences - correlations - in time ${ }^{1}$.

It has been observed that two models of metabolism are possible. In the first one metabolic risk increases progressively as an increasing function of certain physiological variables

\footnotetext{
${ }^{1}$ Networks that consider links associated with correlations in time have also been considered in Ivanov et al. (2017) and Lin et al. (2020).
}

(Wijndaele et al., 2006; Knell et al., 2018). In the second one, metabolic homeostasis is bimodal, and as such, risk increases significantly only upon exceeding certain thresholds associated with the diagnosis of the pathological state (Stern et al., 2005; Alberti et al., 2009). Therefore, to encompass both possibilities, we created Complex Inference Networks for both employing accessible biomarkers that probe the underlying metabolism.

In the first case, the coupling between two physiological variables can be explored through their rate of change in the population. Here, a monotonic association would be found between those variables that interact directly or indirectly within the physiological network. We tested the physiological variables datasets for normality using the Shapiro-Wilk test and screened them for extreme values. Since the data sets were not normally distributed and had extreme values expected to be real, we selected the Spearman Rank Correlation (Batushansky et al., 2016) as a measure of correlation. We modeled the metabolic physiological network as a continuous association of pairs of variables. For this monotonic correlation model, a correlation matrix was constructed for the 15 chosen physiological parameters (Figure 4). Significant correlations were established at a value of $p<0.001$, indicating that the relation does not support the null hypothesis that the independent and dependent variables are unrelated. The weight of the Spearman's rho correlation was squared in order to obtain only positive values.

For the second case, a pathological states network was constructed using currently accepted thresholds from the literature. Here, cut-off values allow the comparison of the tails of the distributions across age groups. The objective here was to indicate whether the participants within the tail of the distribution of one physiological variable have a greater probability of being also in the tail of the distribution of another physiological variable than would be explained by the prevalence of the pathological states alone. This probability of being in a pathological state $\mathrm{B}$ given that the individual is in a pathological state A was described using the following binomial test:

$$
\varepsilon=\frac{N_{x}(P(c \mid x)-P(c))}{\sqrt{N_{x}(1-P(c)) P(c)}}
$$

This test is not necessarily reciprocal, thus giving a weighted directionality to the relationship. If a pathological state is probably the origin of another, their $\varepsilon$ value would be expected to be high in that direction, while it could be low in the opposite one. For this binomial test the null hypothesis is that the probability of presenting condition $\mathrm{C}$ is not affected by having condition $\mathrm{X}$. The statistical significance, $\varepsilon$, is a measure of the extent to which the null hypothesis is verified by the data. In the circumstance, which is valid here, where the binomial distribution can be approximated by a normal distribution, $\varepsilon>1.96$ corresponds to the standard 95\% confidence interval (Easton et al., 2014). As the pathological states network is based upon thresholds accepted by medical consensus, this network adheres well to the known progression of MetS. However, the employment of cutoff values for asserting associations between states may result in an association toward the most sensitive, low thresholds. 
Exceedingly low thresholds can make pathological states seem more prevalent and bias the direction of $\varepsilon$ (Easton et al., 2019). In consequence, care was taken for the selection of thresholds consistent with the preventive scope of MetS.

In summary, for the first case, physiological variables are monotonically correlated along all their biologically plausible spectrum. In this scenario the associations between parameters are present even at healthy values and represent a continuum. For the second case, pathological states are best regarded as binomial. Upon reaching a threshold, the association between these states either appears or increases significantly. This second model resembles the current interpretation of MetS, as it requires a co-occurrence higher than would be expected by chance and contemplates cutoff values as all or nothing states (Alberti et al., 2009). Finally, we used groups of individuals of different ages in order to explore the progressive changes that occur during the aging process and which result in an increasing prevalence of MetS. From the systems biology perspective, the network structure is a direct result of the coordination, or lack thereof, of components that are linked by homeostatic feedback (Goldstein, 2019).

\section{Network Construction and Statistical Analysis}

For the construction of our considered networks we used correlation matrices of physiological variables and pathological states. These matrices were interpreted as weighted adjacency matrices, where adjacency is represented by the Spearman rhos or the $\varepsilon$ values between each pair of metabolic components. The resulting matrices were weighted and undirected for the Pearson correlation matrix and weighted and directed in the case of $\varepsilon$ values (Figure 4). For the construction of the Spearman correlation matrix, data-set normality testing, linear regression and chi-squared tests for trends were all done with Prism 8.1.2(277), GraphPad Software, La Jolla, CA, United States, www.graphpad.com. For the network construction RStudio, an $\mathrm{R}$ language programming suite and igraph package (Csárdi et al., 2016; R Core Team, 2020; RStudio Team, 2020).

Nodes within a network can be ranked according to several centrality definitions that fall into two main groups, radial measures and medial measures. Inferring causality exclusively from centrality within networks requires caution, although eigencentrality has been found to be the best centrality measurement for this purpose, especially for small networks with less than 30 nodes (Dablander and Hinne, 2019). Therefore, we selected eigenvector for undirected networks and hub score for directed networks as radial measures. For medial measures we decided to use flow betweenness. These centrality values allow for a direct comparison of either the influence of nodes (radial measure) or gatekeeping (medial measure) within the network (Borgatti and Everett, 2006). Eigencentrality corresponds to the value of the first eigenvector of the graph adjacency matrix and was interpreted as a measure of influence within the undirected networks. These values were obtained using the evcent function from the SNA package (Katz, 1953; Butts, 2019). For directed networks, hub score and authority score, are a better way of representing influence as these measures takes into account the directionality of the links. Hub scores are defined as the principal eigenvectors of $A^{*} t(A)$, where $A$ is the adjacency matrix of the network. These values were calculated with the hub_score function from the igraph package (Kleinberg, 1998). Flow betweenness was used as a measurement of intermediation within the network. Flow betweenness was calculated using the flowbet function from the SNA package (Koschützki and Schreiber, 2008). In order to test if the eigencentrality and flow betweenness values obtained would be seen in a random graph with the same number of vertices, edges or dyads, univariate conditionally uniform graph tests (CUG test) were employed with the cug.test function from the SNA package.

Networks can contain subgraphs, subsets of vertices with a specific set of edges connecting them within the original graph, that are of particular relevance (Aittokallio and Schwikowski, 2006). We sought two particular subgraphs within our models: First, the graph corresponding to those variables associated with the current definition of MetS, and second, the largest clique within the graph. As there may be more than one combination of nodes that result in a largest clique, we registered the number of times each node appeared within a possible largest clique. These maximally connected subgraphs - largest cliques - were identified using the largest_cliques function of the igraph package (Eppstein et al., 2010). Largest clique and current MetS variables were highlighted as subgraphs, along with the graph diameter.

The largest clique is the biggest, maximally connected subgraph of a graph and contains vertices such that each vertex is connected with every other vertex of the clique. This gives an idea of which vertices go hand in hand in each network (Pavlopoulos et al., 2011). On the other hand, a cluster, as defined using a suitable clustering algorithm, is a group of vertices within a graph that are more densely connected to one another than to other vertices (Csárdi et al., 2016). There are several alternative algorithms for discovering communities of vertices within graphs. For community detection within the networks we used two different algorithms. For the Pearson model, the Louvain algorithm was employed as a heuristic method based on modularity optimization, with the cluster_louvain function from the igraph package (Blondel et al., 2008). In the $\varepsilon$ model, the spinglass community algorithm selects those nodes with the greatest probability to be found in the same state concurrently, with the cluster_spinglass function from the igraph package (Reichardt and Bornholdt, 2006). These two approaches to identifying related biomarkers are complementary - clustering strategies maximize the modularity of the network, while largestclique identification maximizes the transitivity of the largest possible subgraph.

Topological properties were assessed as follows: Density, reciprocity and characteristic path length of the networks were calculated using the igraph package (Faust and Wasserman, 1994; West, 1996; Freeman, 1979). For the calculation of the weighted transitivity and the clustering coefficient in directed and undirected weighted networks the DirectedClustering package was employed (Barrat et al., 2004; Onnela et al., 2005; Fagiolo, 2007; Clemente and Grassi, 2018). The Small world index, as calculated by qgraph, was used as a summary metric of the 
network topology (Watts and Strogatz, 1998). CUG tests were also performed for network density, reciprocity, transitivity and characteristic path length. A glossary of specialized terms is provided in Table 5.

\section{DATA AVAILABILITY STATEMENT}

The original contributions presented in the study are publicly available. This data can be found here: https://www.c3.unam.mx/ health/.

\section{ETHICS STATEMENT}

The studies involving human participants were reviewed and approved by the Comité de Ética en Investigación de la Universidad Nacional Autónoma de México, Facultad de Medicina, under project FM/DI/023/2014. The patients/participants provided their written informed consent to participate in this study.

\section{AUTHOR CONTRIBUTIONS}

CS designed the project and obtained the funding. AB-M conceived the work and implemented the network modeling with JE and AR. JE and AR performed all the statistical and network analysis. AB-M, RM-T, AR-C, and LC contributed with the

\section{REFERENCES}

Aittokallio, T., and Schwikowski, B. (2006). Graph-based methods for analysing networks in cell biology. Brief. Bioinform. 7, 243-255. doi: 10.1093/bib/bbl022

Alberti, K. G. M. M., Eckel, R. H., Grundy, S. M., Zimmet, P. Z., Cleeman, J. I., Donato, K. A., et al. (2009). Harmonizing the metabolic syndrome: a joint interim statement of the international diabetes federation task force on epidemiology and prevention; National heart, lung, and blood institute; American heart association; World heart federation; International. Circulation 120, 1640-1645. doi: 10.1161/CIRCULATIONAHA.109.192644

Almeda-Valdes, P., Aguilar-Salinas, C. A., Uribe, M., Canizales-Quinteros, S., and Méndez-Sánchez, N. (2016). Impact of anthropometric cut-off values in determining the prevalence of metabolic alterations. Eur. J. Clin. Invest. 46, 940-946. doi: 10.1111/eci.12672

American Diabetes, and Association. (2020). 2. classification and diagnosis of diabetes: standards of medical care in diabetes-2020. Diabetes Care 43(Suppl. 1), S14-S31. doi: 10.2337/dc20-S002

Barabási, A.-L., Dezsõ, Z., Ravasz, E., Yook, S., and Oltvai, Z. (2003). "Scale-free and hierarchical structures in complex networks," in AIP Conference Proceedings, Vol. 661, College Park, MA: AIP Publishing, 1-16. doi: 10.1063/1.1571285

Barrat, A., Barthélemy, M., Pastor-Satorras, R., and Vespignani, A. (2004). The architecture of complex weighted networks. Proc. Natl. Acad. Sci. U.S.A. 101, 3747-3752. doi: 10.1073/pnas.0400087101

Batushansky, A., Toubiana, D., and Fait, A. (2016). Correlation-based network generation, visualization, and analysis as a powerful tool in biological studies: a case study in cancer cell metabolism. BioMed Res. Int. 2016, 1-9. doi: 10.1155/ 2016/8313272

Blondel, V. D., Guillaume, J.-L. L., Lambiotte, R., and Lefebvre, E. (2008). Fast unfolding of communities in large networks. J. Stat. Mech. 2008, 1-12. doi: 10.1088/1742-5468/2008/10/P10008

Borgatti, S. P., and Everett, M. G. (2006). A Graph-theoretic perspective on centrality. Soc. Netw. 28, 466-484. doi: 10.1016/J.SOCNET.2005.11.005 acquisition and the medical interpretation of the data. All authors contributed with the manuscript revision, read and approved the submitted version.

\section{FUNDING}

This work was partially supported by the CONACyT through the Fronteras grants FC-2015-2/1093 and Universidad Nacional Autónoma de México through DGAPA Programa de Apoyo a Proyectos de Investigación e Innovación Tecnológica (PAPIT) IG101520, AV100120, IN113619, and PAPIME PE103519. We also acknowledge support from SECTEI CDMX grant SECIT/093/2018 and a donation from Academic Relations, Microsoft Corporation.

\section{ACKNOWLEDGMENTS}

AB-M was a doctoral student from the Programa de Doctorado en Ciencias Biomédicas, Universidad Nacional Autónoma de México (UNAM) and received fellowship 596756 from CONACYT. We thank the Instituto Nacional de Ciencias Médicas y Nutrición "Salvador Zubirán” and the Hospital Juárez de México, along with David Lara Villalón and the clinical laboratory teams, for the sample processing described in the laboratory procedures. The pre-print of this work is available at doi: 10.1101/2020.08.05.20168997.

Broido, A. D., and Clauset, A. (2019). Scale-free networks are rare. Nat. Commun. 10, 1-10. doi: 10.1038/s41467-019-08746-5

Butts, C. T. (2019). sna: Tools for Social Network Analysis. Avaliable at: https: //cran.r-project.org/package=sna (accessed December 10, 2019).

Chan, S. Y., and Loscalzo, J. (2012). The emerging paradigm of network medicine in the study of human disease. Circ. Res. 111, 359-374. doi: 10.1161/ CIRCRESAHA.111.258541

Chen, P., Li, Y., Liu, X., Liu, R., and Chen, L. (2017). Detecting the tipping points in a three-state model of complex diseases by temporal differential networks. J. Transl. Med. 15:217. doi: 10.1186/s12967-017-1320-7

Chiu, Y. F., Hsu, C. C., Chiu, T. H. T., Lee, C. Y., Liu, T. T., Tsao, C. K., et al. (2015). Cross-sectional and longitudinal comparisons of metabolic profiles between vegetarian and non-vegetarian subjects: a matched cohort study. Br. J. Nutr. 114, 1313-1320. doi: 10.1017/S00071145150 02937

Clemente, G. P., and Grassi, R. (2018). Directed clustering in weighted networks: a new perspective. Chaos Solitons Fractals 107, 26-38. doi: 10.1016/j.chaos.2017. 12.007

Csárdi, G., Nepusz, T., and Airoldi, E. M. (2016). Statistical Network Analysis with Igraph. Berlin: Springer.

Dablander, F., and Hinne, M. (2019). Node centrality measures are a poor substitute for causal inference. Sci. Rep. 9:6846. doi: 10.1038/s41598-01943033-9

Easton, J. F., Robles-Cabrera, A., Fossion, R., Rivera, A. L., and Stephens, C. R. (2019). Thoughts on the use of standard cut-off values for physiological health indicators. AIP Conf. Proc. 2090:50006. doi: 10.1063/1.5095921

Easton, J. F., Stephens, C. R., and Angelova, M. (2014). Risk factors and prediction of very short term versus short/intermediate term post-stroke mortality: a data mining approach. Comput. Biol. Med. 54, 199-210. doi: 10.1016/J. COMPBIOMED.2014.09.003

Enzi, G., Busetto, L., Inelmen, E. M., Coin, A., and Sergi, G. (2003). Historical perspective: visceral obesity and related comorbidity in Joannes Baptista 
Morgagni's 'De Sedibus et Causis Morborum per Anatomen Indagata.'. Int. J. Obesity 27, 534-535. doi: 10.1038/sj.ijo.0802268

Eppstein, D., Löffler, M., and Strash, D. (2010). Listing all maximal cliques in sparse graphs in near-optimal time. Algorithms Comput. 6506, 403-414. doi: 10.1007/978-3-642-17517-6_36

Esteghamati, A., Ashraf, H., Esteghamati, A.-R., Meysamie, A., Khalilzadeh, O., Nakhjavani, M., et al. (2009). Optimal threshold of homeostasis model assessment for insulin resistance in an Iranian population: the implication of metabolic syndrome to detect insulin resistance. Diabetes Res. Clin. Pract. 84, 279-287. doi: 10.1016/j.diabres.2009.03.005

Fagiolo, G. (2007). Clustering in complex directed networks. Phys. Rev. E Stat. Nonlin. Soft Matter Phys. 76:026107. doi: 10.1103/PhysRevE.76.026107

Faust, K., and Wasserman, S. (1994). "Notation for social network data," in Social Network Analysis: Methods and Applications, eds K. Faust and S. Wasserman (Cambridge: Cambridge University Press), 69-91. doi: 10.1017/ CBO9780511815478.004

Fossion, R., Rivera, A. L., and Estañol, B. (2018). A physicist's view of homeostasis: how time series of continuous monitoring reflect the function of physiological variables in regulatory mechanisms. Physiol. Meas. 39:084007. doi: 10.1088/ 1361-6579/aad8db

Fossion, R., Stephens, C. R., García-Pelagio, K. P., and García-Iglesias, L. (2017). "Data mining and time-series analysis as two complementary approaches to study body temperature in obesity," in ACM International Conference Proceeding Series, Vol. Part F128634, New York, NY: Association for Computing Machinery, 190-194. doi: 10.1145/3079452.3079504

Freeman, L. C. (1979). Centrality in social networks I: conceptual clarification. Soc. Netw.

Goh, K.-I., Cusick, M. E., Valle, D., Childs, B., Vidal, M., and Szló Barabá, A.L. (2007). The human disease network. Proc. Natl. Acad. Sci. U.S.A. 104, 8685-8690.

Goldstein, D. S. (2019). How does homeostasis happen? Integrative physiological, systems biological, and evolutionary perspectives. Am. J. Physiol. Regul. Integr. Comp. Physiol. 316, R301-R317. doi: 10.1152/ajpregu.00396.2018

Gu, T., Zhou, W., Sun, J., Wang, J., Zhu, D., and Bi, Y. (2018). Gender and age differences in lipid profile among Chinese Adults in Nanjing: a retrospective study of over 230,000 individuals from 2009 to 2015. Exp. Clin. Endocrinol. Diabetes 126, 429-436. doi: 10.1055/s-0043-117417

Haring, R., Rosvall, M., Völker, U., Völzke, H., Kroemer, H., Nauck, M., et al. (2012). A Network-based approach to visualize prevalence and progression of metabolic syndrome components. PLoS One 7:e39461. doi: 10.1371/journal. pone. 0039461

Hildrum, B., Mykletun, A., Hole, T., Midthjell, K., and Dahl, A. A. (2007). Agespecific prevalence of the metabolic syndrome defined by the international diabetes federation and the national cholesterol education program: the norwegian HUNT 2 study. BMC Public Health 7:220. doi: 10.1186/1471-24587-220

Hilgetag, C. C., and Goulas, A. (2016). Is the brain really a small-world network? Brain Struct. Funct. 221, 2361-2366. doi: 10.1007/s00429-015-1035-6

Hofer, S. M., and Sliwinski, M. J. (2001). Understanding ageing. Gerontology 47, 341-352. doi: 10.1159/000052825

Huang, P. L. (2009). A comprehensive definition for metabolic syndrome. Dis. Models Mech. 2, 231-237. doi: 10.1242/dmm.001180

Ivanov, P. C., Liu, K. K. L., Lin, A., and Bartsch, R. P. (2017). "Network physiology: from neural plasticity to organ network interactions," in Springer Proceedings in Physics, Vol. 191, Berlin: Springer Science and Business Media, LLC, 145-165. doi: 10.1007/978-3-319-47810-4_12

Jansson, L. (2020). Network explanations and explanatory directionality. Philos. Trans. R. Soc. B Biol. Sci. 375:20190318. doi: 10.1098/rstb. 2019.0318

Kahn, R. (2007). Metabolic syndrome: is it a syndrome? Does it matter? Circulation 115, 1806-1810. doi: 10.1161/CIRCULATIONAHA.106.658336 discussion 1811.

Kahn, R., Buse, J., Ferrannini, E., Stern, M., American Diabetes Association, and European Association for the Study of Diabetes (2005). The metabolic syndrome: time for a critical appraisal: joint statement from the American Diabetes Association and the European Association for the Study of Diabetes. Diabetes Care 28, 2289-2304. doi: 10.2337/DIACARE.28.9.2289
Kanbay, M., Jensen, T., Solak, Y., Le, M., Roncal-Jimenez, C., Rivard, C., et al. (2016). Uric acid in metabolic syndrome: from an innocent bystander to a central player. Eur. J. Int. Med. 29, 3-8. doi: 10.1016/j.ejim.2015.11.026

Katz, L. (1953). A new status index derived from sociometric analysis. Psychometrika 18, 39-43. doi: 10.1007/BF02289026

Khanna, D., Fitzgerald, J. D., Khanna, P. P., Bae, S., Singh, M. K., Neogi, T., et al. (2012). 2012 American College of Rheumatology Guidelines for Management of Gout. Part 1: systematic nonpharmacologic and pharmacologic therapeutic approaches to hyperuricemia. Arthritis Care Res. 64, 1431-1446. doi: 10.1002/ acr.21772

Kitano, H., Oda, K., Kimura, T., Matsuoka, Y., Csete, M., Doyle, J., et al. (2004). Metabolic syndrome and robustness tradeoffs. Diabetes 53(Suppl. 3), S6-S15. doi: 10.2337/diabetes.53.suppl_3.S6

Kleinberg, J. M. (1998). "Authoritative sources in a hyperlinked environment," in Proceedings of the Nineth ACM-SIAM Symposium on Discrete Algorithms, 45, 604-632. Available online at: www.harvard.edu

Knell, G., Li, Q., Pettee Gabriel, K., and Shuval, K. (2018). Long-term weight loss and Metabolic Health in adults concerned with maintaining or losing weight: findings from NHANES. Mayo Clin. Proc. 93, 1611-1616. doi: 10.1016/ j.mayocp.2018.04.018

Koschützki, D., and Schreiber, F. (2008). Centrality analysis methods for biological networks and their application to gene regulatory networks. Gene Regul. Syst. Biol. 2, 193-201.

Leatherdale, S. T. (2015). An examination of the co-occurrence of modifiable risk factors associated with chronic disease among youth in the COMPASS study. Cancer Causes Control 26, 519-528. doi: 10.1007/s10552-015-0529-0

Leventhal, A. M., Huh, J., and Dunton, G. F. (2014). Clustering of modifiable biobehavioral risk factors for chronic disease in US adults: a latent class analysis. Perspect. Public Health 134, 331-338. doi: 10.1177/1757913913495780

Levin, A., Stevens, P. E., Bilous, R. W., Coresh, J., De Francisco, A. L. M., De Jong, P. E., et al. (2013). Kidney disease: Improving global outcomes (KDIGO) CKD work group. KDIGO 2012 clinical practice guideline for the evaluation and management of chronic kidney disease. Kidney Int. Suppl. . 3, 1-150. doi: 10.1038/kisup. 2012.73

Lin, A., Liu, K. K. L., Bartsch, R. P., and Ivanov, P. C. (2020). Dynamic network interactions among distinct brain rhythms as a hallmark of physiologic state and function. Commun. Biol. 3, 1-11. doi: 10.1038/s42003-020-0878-4

Lusis, A. J., Attie, A. D., and Reue, K. (2008). Metabolic syndrome: from epidemiology to systems biology. Nat. Rev. Genet. 9, 819-830. doi: 10.1038/ nrg2468

Mach, F., Baigent, C., Catapano, A. L., Koskinas, K. C., Casula, M., Badimon, L., et al. (2019). 2019 ESC/EAS Guidelines for the management of dyslipidaemias: lipid modification to reduce cardiovascular risk. Eur. Heart J. 41, 111-188. doi: 10.1093/eurheartj/ehz455

Merico, D., Gfeller, D., and Bader, G. D. (2009). How to visually interpret biological data using networks. Nat. Biotechnol. 27, 921-924. doi: 10.1038/nbt.1567

O'Neill, S., and O'Driscoll, L. (2015). Metabolic syndrome: a closer look at the growing epidemic and its associated pathologies. Obesity Rev. 16, 1-12. doi: 10.1111/obr.12229

Onnela, J. P., Saramäki, J., Kertész, J., and Kaski, K. (2005). Intensity and coherence of motifs in weighted complex networks. Phys. Rev. E Stat. NonlinearSoft. Matter Phys. 71:065103. doi: 10.1103/PhysRevE.71. 065103

Parikh, R., and Mohan, V. (2012). Changing definitions of metabolic syndrome. Indian J. Endocrinol. Metab. 16, 7-12. doi: 10.4103/2230-8210.91175

Pavlopoulos, G. A., Secrier, M., Moschopoulos, C. N., Soldatos, T. G., Kossida, S., Aerts, J., et al. (2011). Using graph theory to analyze biological networks. BioData Mining 4:10. doi: 10.1186/1756-0381-4-10

R Core Team (2020). R: A Language and Environment for Statistical Computing. Viena: R Foundation for Statistical Computing.

Reaven, G. M. (1993). Role of insulin resistance in human disease (syndrome x): an expanded definition. Annu. Rev. Med. 44, 121-131. doi: 10.1146/annurev.me. 44.020193.001005

Reichardt, J., and Bornholdt, S. (2006). Statistical mechanics of community detection. Phys. Rev. E 74:016110. doi: 10.1103/PhysRevE.74.016110

RStudio Team (2020). RStudio: Integrated Development for R. PBC. Boston, MA: RStudio. 
Sattar, N. (2008). Why metabolic syndrome criteria have not made prime time: a view from the clinic. Int. J. Obesity 32(Suppl. 2), S30-S34. doi: 10.1038/ijo. 2008.33

Song, C., Havlin, S., and Makse, H. A. (2005). Self-similarity of complex networks. Nature 433, 392-395. doi: 10.1038/nature03248

Stephens, C. R., Easton, J. F., Robles-Cabrera, A., Fossion, R. Y. M., De La Cruz, L., Martinez-Tapia, R., et al. (2020). The impact of education and age on metabolic disorders. Front. Public Health 8:180. doi: 10.3389/FPUBH.2020.00180

Stephens, C. R., Heau, J. G., González, C., Ibarra-Cerdeña, C. N., Sánchez-Cordero, V., and González-Salazar, C. (2009). Using biotic interaction networks for prediction in biodiversity and emerging diseases. PLoS One 4:e5725. doi: 10. 1371/journal.pone.0005725

Stephens, C. R., Sierra Alcocer, R., and González Salazar, C. (2018). Complex inference networks: a new tool for spatial modelling. Discontinu. Nonlin. Compl. 7, 383-396. doi: 10.5890/DNC.2018.12.003

Stern, S. E., Williams, K., Ferrannini, E., DeFronzo, R. A., Bogardus, C., and Stern, M. P. (2005). Identification of individuals with insulin resistance using routine clinical measurements. Diabetes 54, 333-339. doi: 10.2337/diabetes.54.2.333

Sun, L., Yu, Y., Huang, T., An, P., Yu, D., Yu, Z., et al. (2012). Associations between ionomic profile and metabolic abnormalities in human population. PLoS One 7:e38845. doi: 10.1371/journal.pone. 0038845

Sund-Levander, M., Forsberg, C., and Wahren, L. K. (2002). Normal oral, rectal, tympanic and axillary body temperature in adult men and women: a systematic literature review. Scand. J. Car. Sci. 16, 122-128. doi: 10.1046/j.1471-6712.2002. 00069.x

Toledo-Roy, J. C., Rivera, A. L., and Frank, A. (2019). "Symmetry, criticality and complex systems," in Symmetries and Order: Algebraic Methods in Many Body Systems: A Symposium in Celebration of the Career of Professor Francesco Iachello, Vol. 2150, (College Park, MA: AIP Publishing), 020014. doi: 10.1063/ 1.5124586

Tyagi, A., and Aeddula, N. R. (2019). Azotemia. Treasure Island, FL.: StatPearls Publishing.

Vassallo, P., Driver, S. L., and Stone, N. J. (2016). Metabolic syndrome: an evolving clinical construct. Prog. Cardiovasc. Dis. 59, 172-177. doi: 10.1016/j.pcad.2016. 07.012
Vona, R., Gambardella, L., Cittadini, C., Straface, E., and Pietraforte, D. (2019). Biomarkers of oxidative stress in metabolic syndrome and associated diseases. Oxid. Med. Cell. Longev. 2019, 1-19. doi: 10.1155/2019/8267234

Wallace, T. M., Levy, J. C., and Matthews, D. R. (2004). Use and Abuse of HOMA Modeling. Diabetes Care 27, 1487-1495. doi: 10.2337/diacare.27.6.1487

Watts, D. J., and Strogatz, S. H. (1998). Collective dynamics of 'small-world9 networks. Nature 393, 440-442. doi: 10.1038/30918

West, D. B. (1996). An Introduction to Graph Theory. Upper Saddle River, NJ: Prentice-Hall.

Whelton, P. K., Carey, R. M., Aronow, W. S., Casey, D. E., Collins, K. J., Dennison Himmelfarb, C., et al. (2018). 2017 ACC / AHA / AAPA / ABC / ACPM / AGS / APhA / ASH / ASPC / NMA / PCNA guideline for the prevention, detection, evaluation, and management of high blood pressure in adults: executive summary. J. Am. Coll. Cardiol. 71, 2199-2269. doi: 10.1016/j.jacc. 2017.11.005

Wijndaele, K., Beunen, G., Duvigneaud, N., Matton, L., Duquet, W., Thomis, M., et al. (2006). A continuous metabolic syndrome risk score: utility for epidemiological analyses [6]. Diabetes Care 29:2329. doi: 10.2337/dc06-1341

World Health Organization [WHO] (1995). Physical Status: The Use and Interpretation of Anthropometry. Report of a WHO Expert Committee. Switzerland: World Health Organization technical report series, 854.

Xu, H., Li, X., Adams, H., Kubena, K., and Guo, S. (2018). Etiology of metabolic syndrome and dietary intervention. Int. J. Mol. Sci. 20:128. doi: 10.3390/ ijms20010128

Conflict of Interest: The authors declare that the research was conducted in the absence of any commercial or financial relationships that could be construed as a potential conflict of interest.

Copyright (c) 2020 Barajas-Martínez, Easton, Rivera, Martínez-Tapia, de la Cruz, Robles-Cabrera and Stephens. This is an open-access article distributed under the terms of the Creative Commons Attribution License (CC BY). The use, distribution or reproduction in other forums is permitted, provided the original author(s) and the copyright owner(s) are credited and that the original publication in this journal is cited, in accordance with accepted academic practice. No use, distribution or reproduction is permitted which does not comply with these terms. 